\title{
PREFACE
}

\section{Quest for Quark Gluon Plasma}

"International conference on Matter at Extreme Conditions" (ICMEC 2014) was held at Bose Institute during January 15-17, 2014 in collaboration with Saha Institute of Nuclear Physics, Variable Energy Cyclotron Centre and University of Calcutta. The main aim of this conference was to discuss the evolution of our understanding of matter at extreme conditions of high temperatures and/ or high densities and to discuss the present status and future directions, especially for the benefit of students and young practitioners.

Physics of strongly interacting matter, as we understand today, perhaps took its first step with the classification of hadrons through the SU(3) flavour symmetry proposed independently by Murray GellMann and Y. Ne'eman in early nineteen sixties. This idea finally led to quark model proposed independently by Gell-Mann and George Zweig. The existence of quark became an experimental reality through the discovery of $\mathrm{W}^{-}$along with the results of deep inelastic scattering experiments at SLAC. Concept of "Colour" quantum number was introduced by Gell-Mann and Fritzsch in early seventies. All the hadrons are colour singlet; SU(3) colour symmetry being an exact symmetry. This colour group was interpreted as gauge group with gluons as the coloured gauge bosons. Unlike photons, the gauge bosons of U(1) gauge group, gluons, have self-interaction, which leads to the reduction of coupling constant at higher energies - the concept of asymptotic freedom as discovered by David Gross and Frank Wilczek and independently by David Politzer.

Soon it was realized that the idea of asymptotic freedom, i.e., the fact that the interaction between quarks, which is very strong at large separation/low energy, becomes weaker with decreasing separation or increasing energy, has interesting consequences. Though in the present era with vanishing temperatures and densities, the Universe consists of normal hadronic matter, the early Universe with extreme temperatures must have existed in the form of quark gluon matter or quark gluon plasma (QGP), albeit for a short time scale. Moreover, presently the core of compact objects like Neutron stars, where densities may be as large as 8-10 times the normal nuclear matter densities, may also consist of quark matter.

In addition to the above scenario in nature, it was also realized during the early days itself, that the collision of heavy nuclei at high energies might create suitable environment of high temperatures and densities, necessary for the creation of QGP. Along with the development of new colliders with increasing energy, starting from AGS to SPS to RHIC and then LHC, the physical understanding of QGP has also evolved and progressed quite well.

The idea of phase transition between hadronic and quark matter allows one to use the concept of statistical mechanics for the study of the properties 
of QGP. Initially, during late seventies, QGP was visualized as the gas of dressed quarks and gluons which interact relatively weakly with each other. However, the huge amount of data produced in the experiments has not only changed our perception altogether, it has given rise to new ideas and concepts as well. For example, the collective behaviour of system produced in the collision became apparent from the early days of RHIC itself. This implies that the effective interaction in QGP is in fact much stronger than expected on the basis of the perturbation theory. Predictions based on Ideal hydrodynamics were found to be in better agreement with the data. Instead of a weakly interacting gas, QGP is now perceived to be an almost ideal fluid with low viscosity.
With the participation of more countries in the International collaborations, QGP community has expanded. New facilities like FAIR have been planned to shed light on the less explored realm of high baryon densities. India has been a part of these efforts since early 1990s. Various research institutes as well as universities have become regular participants in this International quest for understanding the physics of QGP. Indian QGP community has also grown considerably over the time. The present proceedings showcase the efforts of Indian community along with developments at international level. The conference discussed wide variety of topics covering both physical as well as astrophysical aspects of matter at extreme conditions. Reviews by the experts covered the progress in our knowledge in different areas of interest. In addition, there were presentations by

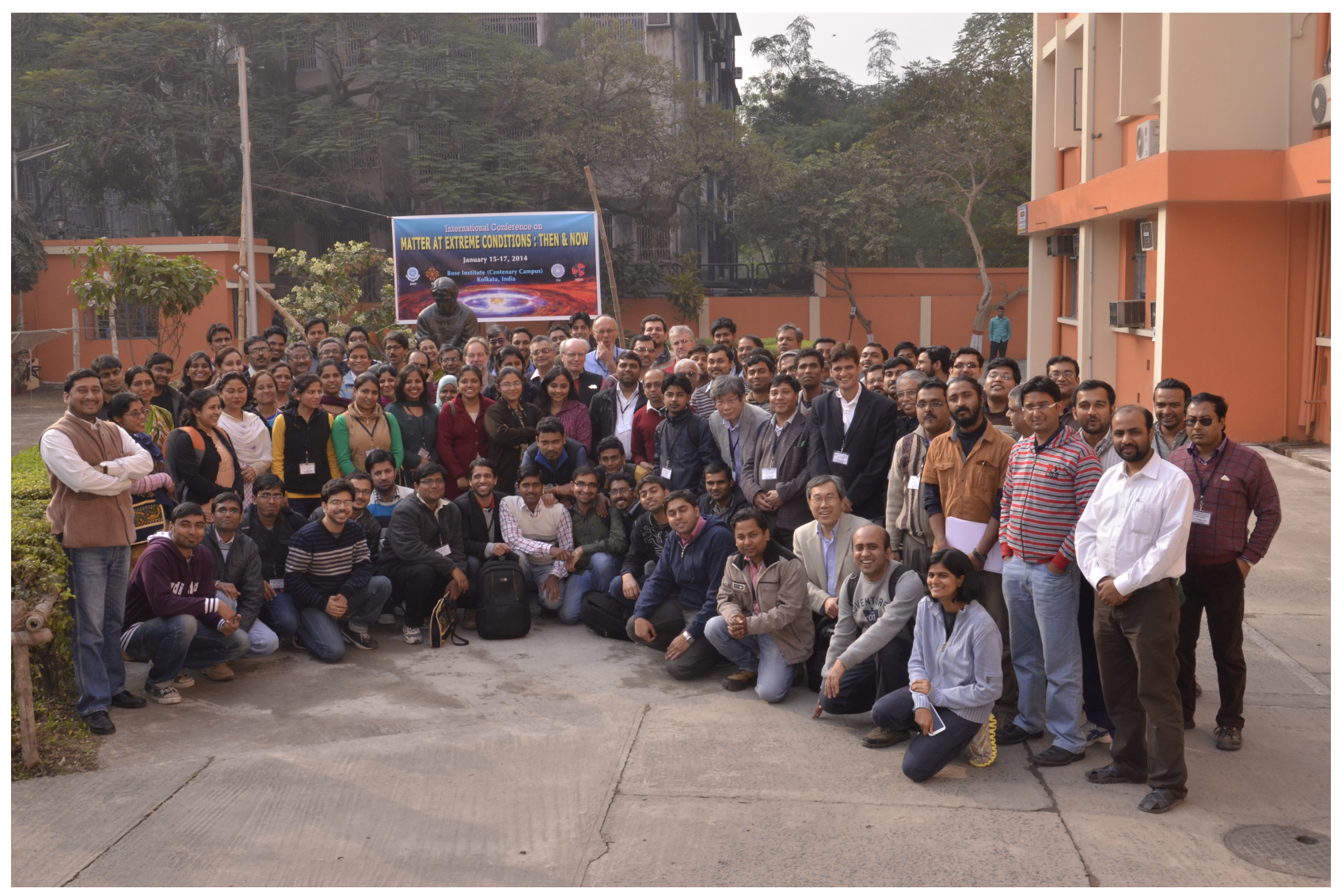

Participants at ICMEC-2014 
students and young researchers covering experimental results and theoretical understanding.

The papers included in this special issue are based on presentations at this meeting. We thank all the contributing authors for their cooperation. We sincerely hope that this issue will be useful to the community.

This conference also marked an important occasion. Prof. Sibaji Raha, present Director of Bose Institute, has been associated with QGP community from the very beginning. He has contributed significantly to this area of research. Moreover, many physicists, of younger generations have collaborated with him and have benefited with his association. A FEST colloquium was organized on the occasion of Prof. Raha's 60th birthday on January 15, 2014 to honour him for his enormous contributions. This FEST colloquium, organized in the auditorium of the main campus of Bose Institute, was attended by most of the conference participants as well as some of the old friends of Prof. Raha. Several speakers reminisced about their collaboration with Prof. Sibaji Raha. Besides humorous and lighter moments, the participants found it extremely interesting and profitable as these reminiscences gave them a glimpse of the evolution of the subject as well.

We, the organizers, are immensely grateful to the INSA for agreeing to publish these articles as a special issue of the Proceedings of the Indian National Science Academy. We are extremely thankful to Prof. S. C. Lakhotia, Editor-in-Chief, for his constant help and encouragement.

\section{Sanjay K Ghosh} Guest Editor Bose Institute Kolkata 700091 (Email: sanjay@jcbose.ac.in) 
